Injuries are the fourth most common cause of death. Annually about 2,500 Finns die accidentally (Population 5.4 million).

Results The multi-sectoral coordination group has drawn up a national target and action programme for the prevention of home and leisure accident injuries 2014-2020 (http://www.julkari.fi/handle/10024/126217). The programme encompasses 91 actions, for each of which the coordination group has designated bodies responsible for them. In this programme, by home and leisure accident injuries is meant accident injuries other than those occurred at work or in traffic.

The coordination group has defined the most important measures for the following sets of actions: improved safety culture and strengthened safety work, prevention of accident injuries related to the use of medicines, alcohol and drugs, increased equality and in particular improving the safety of vulnerable groups, improved environmental and product safety, and prevention of falling accidents. Specific objectives have been defined for each set of actions.

Conclusion The vision of the present national programme to prevent home and leisure accident injuries is that no one needs to die or be injured as a result of an accident. The objectives of the programme include reaching a good safety level in all environments, $25 \%$ reduction in the number of serious accident injuries by 2025 and allocation of more substantial and permanent resources for accident injury prevention.

\section{ADDRESSING FATAL INJURY IN LOW-MIDDLE INCOME COUNTRIES: THE RESEARCH-POLICY-PRACTICE-CONTEXT NEXUS}

Chebiwot Kipsaina, Joan-Ozanne Smith, Virginia Routley. Department of Forensic Medicine, Monash University, Australia

10.1136/injuryprev-2016-042156.200

Background Despite evidence that injury impedes development in low-middle income countries (LMICs), injury prevention remains low priority in global agendas. Current injury prevention in LMICs is largely based on the Public Health Approach (PHA), with most strategies borrowing high income country interventions based on good injury surveillance systems (ISS).

A study was conducted to investigate the capacity and adequacy of the PHA for injury prevention in LMICs.

Methods Taking data as an indicative key element of the PHA, a systematic review was conducted to assess the utilisation, efficacy and effectiveness of the existing WHO Injury Surveillance Guidelines (2001) in LMICs. Subsequent, LMIC focused fatal injury surveillance guidelines were developed, evaluated by pilot studies conducted in six LMICs for process and case capture effectiveness.

Results The review identified limitations of the WHO 2001 guidelines in LMIC utilisation: mainly short-term studies, ISS not ongoing; single issues addressed; minimum dataset use lacked detail for injury prevention; local capacity not built.The pilot studies showed eligible external cause deaths are poorly captured and reported by the medico-legal system, apparent systemic issues, limited workforce capacity and training in ISS; lack of strong stewardship for fatal injury surveillance. Nonetheless, indicative data on injury deaths was obtained.

Conclusions Complementing the PHA, fatal injury response in LMICs must (i) continue to improve fatal injury data quality to quantify the issue and identify solutions, (ii) evidence gaps should not paralyse progress, rather the 'policy window' opportunity in the new Sustainable Development Goals should be seized, (iii) consider complex contextual and systemic issues in LMIC injury prevention policies. Proposed therefore is a modified model seeking to complement existing approaches by accounting for content, process, practice, policy environment and context for injury prevention in LMICs.

\section{INJURY PREVENTION IN THE WHO SOUTH-EAST ASIA REGION, 2005-2015: FROM POLICY TO PRACTICE}

${ }^{1,2}$ Chamaiparn Santikarn. ${ }^{1}$ Ex-WHO South-East Asia Regional Office (SEARO), India; ${ }^{2}$ WHO Office, Myanmar

10.1136/injuryprev-2016-042156.201

Background Several WHO resolutions on injury prevention were adopted since 1966 and several UN resolutions on road safety were adopted since 2003. The WHO South- East Asia (SEA) Regional committee resolution on accident prevention and trauma care was adopted in 1994. However, in 2005 injury prevention did not progress substantially.

Methods In 2005, WHO South-East Asia Regional Office (SEARO) modified the post description of Regional advisor responsible for injury prevention to be more focused. SEARO injury work plan 2006-7 formed the strategies in promoting injury information, multi-sectoral and inter-country collaborations, experience sharing, and training. In 2008-9 work plan, establishment of an injury management unit in the $\mathrm{MOH}$ was added. Regular regional and national trainings/workshops were organised and supported. In 2010, the Regional resolution: Injury prevention and safety promotion was adopted. It identifies the major causes of injury in the region, endorses existing strategies and calls for a national mechanism at the highest level to enhance national plans/programs. A progress report was requested for 2014. The Global Road Safety Status Surveys and the Decade of Action for Road Safety were coordinated. Regional epidemiological data, the expert group recommendations and the world reports were used for guiding interventions. Resources were mobilised by WHO Geneva to support regional and national activities.

Results By 2015, all countries have national policies/plans. 7 countries have national policies, budgeted plans and a mechanism at the highest level for road safety. 5 countries have budgeted plans for the Decade. 6 countries have budgeted plans to strengthen injury data. 4 MOH's have injury management units. Injury prevention is integrated into undergraduate medical and nursing curriculum and $\mathrm{MCH}$ systems. 2 countries enforce and manufacture standardised child motorcycle helmets. 2 countries have sustained injury surveillance.

Conclusions Progress is seen in the SEA Region from 20052015.

\section{A NEW MODEL FOR TRANSLATING RESEARCH TO POLICY: THE CONSORTIUM FOR EVIDENCE-BASED POLICY}

${ }^{1}$ Shannon Frattaroli, ${ }^{2}$ Joshua Horwitz, ${ }^{3} E$ Beth McGinty, ${ }^{1}$ Andrea C Gielen. 'The Johns Hopkins Centre for Injury Research and Policy, USA; ${ }^{2}$ The Educational Fund to Stop Gun Violence, USA; ${ }^{3}$ The Johns Hopkins Bloomberg School of Public Health, USA

10.1136/injuryprev-2016-042156.202

Background Effective and promising policy interventions exist for many injury problems. There is a need for strategies to 